Research Paper

\title{
A phase II study of chemotherapy in combination with telomerase peptide vaccine (GV1001) as second-line treatment in patients with metastatic colorectal cancer
}

\author{
Sejin Kim ${ }^{1 *}$, Bum Jun Kim ${ }^{1 *}$, Ilhwan Kim², Jung Han Kim¹, Hee Kyung Kim³, Hyewon Ryu ${ }^{4}$, Dae Ro Choi ${ }^{1}$, \\ In Gyu Hwang 5 , Hunho Song ${ }^{1}$, Jung Hye Kwon ${ }^{1}$, Joo Young Jung ${ }^{1}$, Boram Han¹, Dae Young Zang ${ }^{1 凶}$ \\ 1. Division of Hematology-Oncology, Department of Internal Medicine, Hallym University Medical Center, Hallym University College of Medicine, \\ Anyang-si, Republic of Korea. \\ 2. Division of Oncology, Department of Internal Medicine, Haeundae Paik Hospital, Inje University College of Medicine, Busan, Republic of Korea. \\ 3. Department of Internal Medicine, Chungbuk National University College of Medicine, Cheongju, Republic of Korea. \\ 4. Division of Hematology and Oncology, Department of Internal Medicine, Chungnam National University Hospital, Chungnam National University College \\ of Medicine, Daejeon, Republic of Korea. \\ 5. Department of Internal Medicine, Chung-Ang University Hospital, Chung-Ang University College of Medicine, Seoul, Republic of Korea. \\ *These authors contributed equally as first authors.
}

$\triangle$ Corresponding author: Dae Young Zang M.D., Ph.D., Division of Hematology-Oncology, Department of Internal Medicine, Hallym University Medical Center, Hallym University College of Medicine, Anyang-si, Republic of Korea. Tel: +82-31-380-3704; Fax: +82-31-380-1528; E-mail: fhdzang@hallym.or.kr

(c) The author(s). This is an open access article distributed under the terms of the Creative Commons Attribution License (https://creativecommons.org/licenses/by/4.0/). See http://ivyspring.com/terms for full terms and conditions.

Received: 2021.12.22; Accepted: 2022.01.26; Published: 2022.02.14

\begin{abstract}
Background: GV1001 is a human telomerase peptide vaccine that induces a CD4/CD8 T-cell response against cancer cells, thereby affording an immunological anti-tumor effect. Here, we evaluated the efficacy and safety of GVIO0I in combination with chemotherapy in patients with metastatic colorectal cancer who had failed first-line chemotherapy.

Methods: This multicenter, non-randomized, single-arm phase II study recruited recurrent or metastatic colorectal cancer patients with measurable disease who had failed first-line chemotherapy. Patients received GV100I and chemotherapy concomitantly based on a pre-established schedule. Cytotoxic chemotherapy and targeted agents (bevacizumab, cetuximab, or aflibercept) were allowed to be used at the discretion of the investigator. The primary endpoint was the disease control rate; secondary endpoints were the objective response rate, progression-free survival, overall survival, and safety outcomes. The baseline serum eotaxin level (a potential predictive biomarker of GV1001) was analyzed. To determine whether an adequate immune response had been induced, a delayed-type hypersensitivity test and a T-cell proliferation test were performed.
\end{abstract}

Results: From May 13, 2015 to October 13, 2020, 56 patients with recurrent or metastatic colorectal cancer treated in seven hospitals of South Korea were enrolled. The median patient age was 64 years (range, $29-82$ years); $67.9 \%$ were men. Of all patients, $66.1 \%$ had left-side colorectal cancer and the RAS mutation was present in $25 \%$. The disease control rate and the objective response rates were $90.9 \%(95 \%$ confidence interval $[\mathrm{Cl}]: 82.4-99.4 \%)$ and $34.1 \%(95 \% \mathrm{Cl}, 20.1-48.1 \%)$, respectively. The median progression-free survival was 7.1 months $(95 \% \mathrm{Cl}, 5.2-9.1$ months) and the median overall survival was 12.8 months $(95 \% \mathrm{Cl}, 9.9-15.8$ months). The most common all-grade adverse events were neutropenia $(48.2 \%)$, nausea $(26.8 \%)$, neuropathy $(25.0 \%)$, stomatitis $(21.4 \%)$, and diarrhea $(21.4 \%)$. Immune response analysis showed that no patient had positive delayed-type hypersensitivity test results; antigen-specific T-cell proliferation was observed in only $28 \%$ of patients. The baseline eotaxin level was not associated with any efficacy outcome.

Conclusion: Although no clear GV1001-specific immune response was observed, the addition of GV1001 vaccination to chemotherapy was tolerable and associated with modest efficacy outcomes.

Key words: Colorectal neoplasm, cancer vaccines, GV1001 


\section{Introduction}

Colorectal cancer (CRC) is the third most common cancer and the second leading cause of cancer death worldwide [1]. Although the overall mortality of CRC continues to decline if the disease is operable, the survival outcomes of metastatic disease remain dismal. Chemotherapy in combination with targeted monoclonal antibodies has become the main treatment modality for inoperable disease; however, immunotherapy has recently changed the treatment paradigm for metastatic CRCs. After pembrolizumab, an anti-programmed death 1 (PD-1) immune checkpoint inhibitor, exhibited a significant clinical benefit in patients with mismatch repair-deficient (dMMR) CRCs [2], many immune checkpoint inhibitors were investigated. At the 2021 American Society of Clinical Oncology (ASCO) annual meeting, pembrolizumab first-line therapy was reported to be superior to chemotherapy in patients with dMMR CRCs [3]. Anti-cancer vaccination is another type of immunotherapy; many vaccines (including peptide, autologous, and dendritic cell vaccines) have been tested in CRC patients. However, no such vaccine has exhibited a clinical benefit thus far [4-6].

Telomerase (a telomere-repair enzyme) is expressed in $85-90 \%$ of human solid cancers [7]; thus, it is an attractive target for anti-cancer treatment. In normal cells, the telomeric ends of DNA become progressively shortened with repeated cell division; the cells eventually enter replicative senescence [8]. However, cancer cells avoid such senescence, becoming immortal through the reactivation of telomerase. This has a crucial role in the oncogenic transformation of many cancers, including CRCs [9, 10].

GV1001 is a human telomerase, 16-amino acid peptide vaccine derived from the reverse transcriptase subunit. GV1001 induces a CD4/CD8 T-cell response against cancer cells, yielding an immunological anti-tumor effect [11]. After a GV1001-specific immune response and promising efficacy results were obtained in early-stage clinical studies of patients with pancreatic and non-small cell lung cancers $[12,13]$, a large-scale study evaluating the synergistic effects of GV1001 and conventional chemotherapy in pancreatic cancer patients was conducted, but failed to prove the benefit over chemotherapy alone [14]. However, in the subgroup analysis, patients with high baseline eotaxin level were significantly associated with better overall survival (OS) with GV1001 vaccination [15]. At the 2021 ASCO annual meeting, synergistic effects of GV1001 and conventional chemotherapy were reported in pancreatic cancer patients with high eotaxin levels [16]. The median OS significantly improved after GV1001 vaccination plus chemotherapy compared to chemotherapy alone (11.3 months [ $95 \% \mathrm{CI}, 8.6-14.0$ months] vs. 7.5 months [95\% CI, 5.1-10.0 months], $\mathrm{p}=0.021$ ).

GV1001 has also been investigated in advanced melanoma [17], B-cell chronic lymphocytic leukemia [18], cutaneous T-cell lymphoma [19] and has shown modest efficacy outcome with induction of immune response. However, to date, GV1001 has not been investigated in patients with advanced CRCs.

Most (approximately $85 \%$ of patients) CRCs have chromosomal instability (CIN), while other CRCs have a high grade microsatellite instability (MSI) phenotype, and telomere dysfunction may be considered a major driving mechanism of CIN development [10]. Consistent results that increased telomerase activity is associated with tumor progression and poor survival have been reported [20], and these results provides a theoretical background for investigating GV1001, a telomerase peptide vaccine, in patients with advanced CRCs.

In this study, we evaluate the efficacy and safety of GV1001 vaccination in combination with chemotherapy as a second-line treatment for patients with metastatic CRCs.

\section{Methods}

\section{Study design and patient eligibility}

This study was a multicenter, single arm, phase 2 trial done at 7 hospitals in South Korea.

Patients were eligible for this study if they fulfilled all of the following criteria: (1) pathologically confirmed recurrent, or metastatic colorectal cancer patients who failed fist-line chemotherapy (oxaliplatin or irinotecan containing regimen); (2) measurable disease, as defined using version 1.1 of the Response Evaluation Criteria In Solid Tumors (RECIST); (3) age $\geq 19$ years; (4) Eastern Cooperative Oncology Group (ECOG) performance status of 0-2; (5) life expectancy $\geq 12$ weeks; (6) adequate hematological, renal, and hepatic functions, as defined using an absolute neutrophil count of $\geq 1.5 \times$ $10^{9} / \mathrm{L}$, a platelet count of $\geq 100 \times 10^{9} / \mathrm{L}$, serum creatinine levels of $\leq 1.5 \times$ upper limit of normal or creatinine clearance $\geq 50 \mathrm{~mL} / \mathrm{min}$, serum bilirubin $\leq 2$ $\times$ UNL, aspartate aminotransferase and alanine aminotransferase levels of $\leq 2.5 \times$; and (7) willingness to provide informed consent to participate in this study.

Patients were excluded based on the following criteria: (1) other previous or concurrent malignancies within the last 5 years, with the exception of cured basal cell carcinoma of the skin or carcinoma in situ of the uterine cervix; (2) presence of intracerebral 
metastases or meningeal carcinomatosis; (3) other clinically significant comorbid conditions, such as an active infection or severe cardiopulmonary dysfunction; (4) medication that might affect immunocompetence such as long-term steroids or other immunosuppressants for an unrelated condition.

\section{Treatment}

The vaccine GV1001 consists of a synthetic peptide corresponding to the 16 amino acid residue 611 to 626 (EARPALLTSRLRFIPK) of human telomerase reverse transcriptase (hTERT) and is capable of binding to molecules encoded by multiple alleles of all three loci of HLA class II. GV1001 was manufactured by Samsung Pharmacy (Hwasung-si, Korea) and supplied by GemVax \& KAEL (Seongnam-si, Korea).

The selection of second-line chemotherapeutic agents and targeted agents (bevacizumab, cetuximab or aflibercept) depended on the investigator's choice. $0.56 \mathrm{mg}$ of GV1001 was injected intradermally on days $1,3,5$ and 8 during the first cycle of chemotherapy, then once on day 1 of subsequent cycles. GV1001 was diluted with $0.3 \mathrm{ml}$ of $0.9 \%$ normal saline and administered intradermally to the lower abdomen within 6 hours after dilution. This treatment was repeated every 2 weeks until treatment is discontinued due to the subject's request, toxicities, or disease progression.

\section{Serum eotaxin level test}

To determine the relationship between eotaxin level and treatment response, we conducted eotaxin level test in patients who consented to the test. Peripheral blood was collected at the baseline, the first day of $2^{\text {nd }}, 4^{\text {th }}, 7^{\text {th }}, 10^{\text {th }}$ cycles of treatment and analyzed using Bio-Plex ${ }^{\circledR} 200$ systems at the Seoul Clinical Laboratories.

\section{Delayed-type hypersensitivity test}

Delayed-type hypersensitivity (DTH) test was performed to determine whether an immune response has been induced. The test was performed at the baseline and on the first day of $2^{\text {nd }}, 4^{\text {th }}, 7^{\text {th }}$ and $10^{\text {th }}$ cycles of chemotherapy. We continued the test until the result was positive. $0.08 \mathrm{~mL}$ of the remaining solution (Solution A) after preparation for GV1001 injection is diluted in $0.22 \mathrm{~mL}$ of normal saline with a concentration of about $0.7 \mathrm{mg} / \mathrm{ml}$ (Solution B). $0.15 \mathrm{ml}$ of solution $\mathrm{B}$ is extracted and administered intradermally on the opposite lower abdomen within 6 hours after GV1001 injection. If the erythema or induration is more than $5 \mathrm{~mm}$, it is evaluated as positive.

\section{T-cell proliferation test}

Peripheral blood mononuclear cells (PBMCs) were isolated from blood samples before the start of vaccination and on the first day of $2^{\text {nd }}, 4^{\text {th }}, 7^{\text {th }}$, and $10^{\text {th }}$ cycles of chemotherapy to conduct T-cell proliferation test. T-cell proliferation was detected by flow cytometry using carboxy fluorescein diacetate succinimidyl ester (CFSE) (eBioscience 65-0850). After thawing PBMCs in at the end of the cycle, $1 \sim 5 \times 10^{6}$ cells were incubated with $2 \mathrm{mM}$ CFSE at RT for $10 \mathrm{~min}$, washed with ice-cold completed RPMI1640 medium. $1 \times 10^{5}$ CFSE stained cells were seeded in an anti-Human CD3 $(1 \mathrm{mcg} / \mathrm{ml})$ coated 96well culture plate in completed RPMI1640 medium. CFSE labeled PBMCs were stimulated with anti-Human CD28 (1 $\mathrm{mcg} / \mathrm{mL})$, GV1001 peptide $(20 \mathrm{mcg} / \mathrm{mL})$ in the anti-Human CD3 coated 96 well culture plate. PBMCs were incubated at $37{ }^{\circ} \mathrm{C}, 5 \% \mathrm{CO} 2$ for $72 \mathrm{~h}$. Dividing cells were detected by flow cytometry and analyzed using CYTOFLEX software (Beckman).

A positive proliferative $\mathrm{T}$-cell response was defined if one of the following criteria was met: i) a stimulation index (SI) $\geq 2$ (SI was calculated by dividing T-cell population after GV1001 injection by that of the baseline value); ii) the difference in the number of T-cell division before and after GV1001 injection $\geq 1$.

\section{Endpoints}

The primary endpoint was disease control rate (DCR) and secondary endpoints were overall response rate (ORR), progression-free survival (PFS), OS and toxicity profiles. DTH test and T-cell proliferation test was performed to evaluate immune response which was the exploratory endpoint.

\section{Statistical analysis}

According to Simon's optimal two-stage design, 46 patients were required for enrollment to test the null hypothesis that the true DCR is $30 \%$ versus the alternative hypothesis that the true DCR is at least $50 \%$, with two-sided alpha of 0.10 and $90 \%$ power. If 7 patients or more with disease control (complete response + partial response + stable disease) were observed among 22 patients in the first stage, the study was continued with 24 additional patients included. As the drop-out rate was assumed to be $20 \%$, the number of patients necessary for recruitment into the study was calculated to be 57 .

Descriptive statistics were used to summarize the patients' characteristics, tumor responses, and safety events. The Kaplan-Meier method was used to estimate the median PFS and OS. All patients who received at least one cycle of treatment were included in the safety analysis and those who underwent at 
least one response evaluation were defined as modified intent-to-treat (mITT) population and included in the efficacy analysis.

\section{Results}

\section{Patient characteristics}

From May 13, 2015 to October 13, 2020, 56 patients with recurrent or metastatic CRC treated in seven hospitals of South Korea were enrolled. Table 1 shows the baseline characteristics of all patients. The median age was 64 years (range, 29-82 years) and $67.9 \%$ were men. Of all patients, $92.8 \%$ exhibited ECOG performance status $0-1$. The primary tumors were predominantly located in the left side of the colon (left- vs. right-sided, $66.1 \%$ vs. $33.9 \%$ ) and the RAS mutation was present in $25 \%$ of patients.

Table 1. Baseline characteristics of patients (All patients, $n=56$ )

\begin{tabular}{|c|c|}
\hline & Number of patients (\%) \\
\hline \multicolumn{2}{|l|}{ Age, years } \\
\hline Median (range) & $64(29-82)$ \\
\hline \multicolumn{2}{|l|}{ Sex } \\
\hline Male & $38(67.9 \%)$ \\
\hline Female & $18(32.1 \%)$ \\
\hline \multicolumn{2}{|l|}{ ECOG performance status } \\
\hline 0 & $32(57.1 \%)$ \\
\hline 1 & $20(35.7 \%)$ \\
\hline 2 & $4(7.1 \%)$ \\
\hline \multicolumn{2}{|l|}{ Disease status } \\
\hline Initially metastatic & $39(69.6 \%)$ \\
\hline Recurrent & $17(30.4 \%)$ \\
\hline \multicolumn{2}{|l|}{ Site of primary tumor } \\
\hline Ascending colon & $8(14.3 \%)$ \\
\hline Transverse colon & $7(12.5 \%)$ \\
\hline Descending colon & $5(8.9 \%)$ \\
\hline Recto-simoid colon & $32(57.1 \%)$ \\
\hline NA & $4(7.1 \%)$ \\
\hline \multicolumn{2}{|l|}{ Histological differentiation } \\
\hline Well/moderate & $46(82.1 \%)$ \\
\hline Poor/undifferentiated & $8(14.3 \%)$ \\
\hline NA & $2(3.6 \%)$ \\
\hline \multicolumn{2}{|l|}{ Previous first-line treatment } \\
\hline FOLFOX/FOLFIRI + cetuximab & $15(26.8 \%)$ \\
\hline FOLFOX/FOLFIRI + bevacizumab & $21(37.5 \%)$ \\
\hline FOLFOX/FOLFIRI & $20(35.7 \%)$ \\
\hline \multicolumn{2}{|l|}{ RAS mutation } \\
\hline Wild type & $32(57.1 \%)$ \\
\hline Mutant & $14(25.0 \%)$ \\
\hline NA & $10(17.9 \%)$ \\
\hline \multicolumn{2}{|l|}{ Number of metastatic site } \\
\hline $1-2$ & $34(60.7 \%)$ \\
\hline$\geq 3$ & $10(17.9 \%)$ \\
\hline NA & $12(21.4 \%)$ \\
\hline
\end{tabular}

ECOG: Eastern Cooperative Oncology Group; NA: not available; FOLFOX: 5-fluorouracil, leucovorin, and oxaliplatin; FOLFIRI: 5-fluorouracil, leucovorin, and irinotecan.

\section{Efficacy outcomes}

In the first stage of the study, the DCR in the initial 22 patients was $81.8 \%$ (18 of 22); we thus proceeded to the second stage. Of all patients, efficacy analysis was performed on the mITT population; 12 patients were excluded because they received no treatment or did not undergo any tumor assessment. In the mITT population $(\mathrm{n}=44)$, the DCR and ORR were $90.9 \%$ (95\% CI, 82.4-99.4) and 34.1\% (95\% CI, 20.1-48.1), respectively (Table 2). One patient exhibited a complete response (2.3\%). The median PFS was 7.1 months (95\% CI, 5.2-9.1 months) and the median OS was 12.8 months (95\% CI, 9.9-15.8 months) (Figure 1). We performed efficacy analysis according to the baseline eotaxin levels of all available patients $(\mathrm{n}=22)$; patients with lower eotaxin levels $(<80$ $\mathrm{pg} / \mathrm{mL}$ ) showed trends toward better efficacy outcomes (compared to patients with higher eotaxin levels $[\geq 80 \mathrm{pg} / \mathrm{mL}])$ in terms of the DCR $(100.0 \%$ vs. $85.7 \%, \mathrm{p}=0.481)$ and the ORR $(64.3 \%$ vs. $28.6 \%, \mathrm{p}=$ 0.128) (Table 3), but there was no statistical significance.

Table 2. Best overall response (mITT population, $n=44)$

\begin{tabular}{|c|c|}
\hline & mITT population $(n=44)$ : Number of patients $(\%)$ \\
\hline \multicolumn{2}{|l|}{ Best overall response } \\
\hline Complete response (CR) & $1(2.3 \%)$ \\
\hline Partial response (PR) & $14(31.8 \%)$ \\
\hline Stable disease (SD) & $25(56.8 \%)$ \\
\hline Progressive disease (PD) & $4(9.1 \%)$ \\
\hline Objective response rate & $34.1 \%(95 \%$ CI, $20.1-48.1)$ \\
\hline Disease control rate ${ }^{b}$ & $90.9 \%(95 \%$ CI, 82.4-99.4) \\
\hline \multicolumn{2}{|c|}{$\begin{array}{l}\text { a Objective response rate is defined as the proportion of patients with } \mathrm{CR} \text { or PR a } \\
\text { best overall response; }\end{array}$} \\
\hline
\end{tabular}

Table 3. Best overall response by baseline eotaxin level $(n=28)$

\begin{tabular}{llll}
\hline & $\begin{array}{l}\text { Eotaxin high }(\geq 80 \\
\mathrm{pg} / \mathrm{ml})(\mathrm{n}=14)\end{array}$ & $\begin{array}{l}\text { Eotaxin low }(<80 \\
\mathrm{pg} / \mathrm{ml})(\mathrm{n}=14)\end{array}$ & $\begin{array}{l}\mathrm{P} \\
\text { value }\end{array}$ \\
\hline Best overall response & & & \\
Complete response (CR) & $0(0.0 \%)$ & $1(7.2 \%)$ & \\
Partial response (PR) & $4(28.6 \%)$ & $8(57.1 \%)$ & \\
Stable disease (SD) & $8(57.1 \%)$ & $5(35.7 \%)$ & 0.128 \\
Progressive disease (PD) & $2(14.3 \%)$ & $0(0.0 \%)$ & \\
Objective response rate & $28.6 \%$ & $64.3 .0 \%$ & \\
& $(95 \% \mathrm{CI}, 4.9-52.3)$ & $(95 \% \mathrm{CI}, 39.2-89.4)$ & \\
Disease control rate $^{\mathrm{b}}$ & $85.7 \%$ & $100.0 \%$ & \\
& $(95 \% \mathrm{CI}, 67.4-100.0)$ & $(95 \% \mathrm{CI}, 100.0-100.0)$ & \\
\hline
\end{tabular}

a Objective response rate is defined as the proportion of patients with CR or PR as best overall response;

b Disease control rate is $\mathrm{CR}+\mathrm{PR}+\mathrm{SD}$ (including non-CR/non-PD).

CI: confidence interval.

\section{Safety outcomes}

Table 4 lists the treatment-related adverse events. All-grade neutropenia (48.2\%), anemia $(42.9 \%)$, nausea $(26.8 \%)$, neuropathy $(25.0 \%)$, stomatitis (21.4\%), and diarrhea (21.5\%) were common $(\mathrm{n}=56)$. The most common grade $\geq 3$ adverse event was neutropenia (16.1\%).

\section{Prognostic factors}

We performed univariate and multivariate analyses to identify factors potentially prognostic of PFS and OS (Table S1). The multivariate analysis 
included factors with $\mathrm{p}$-values $<0.5$ in the univariate analyses. In the mITT population, two factors were independently associated with a poor PFS in multivariable analysis: age $\geq 65$ years (hazard ratio for PFS, 3.37 [95\% CI, 1.34-8.49], $\mathrm{p}=0.010$ ) and ECOG performance status 1 or 2 (hazard ratio for PFS, 2.6 [95\% CI, 1.01-6.69], $\mathrm{p}=0.048)$.
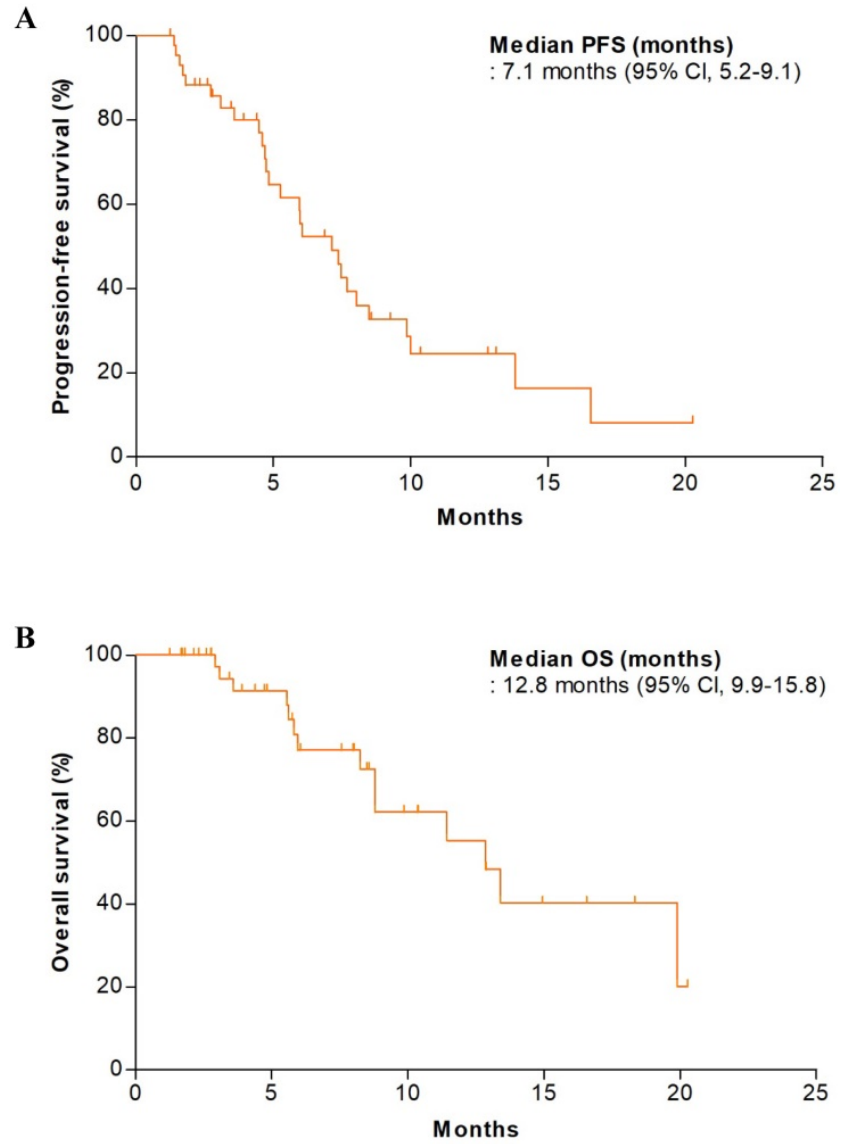

Figure 1. Kaplan-Meier estimates of (A) progression-free survival and (B) overall survival (mITT population, $n=44)$.

\section{DTH and T-cell proliferation tests}

DTH results were available for 20 patients; no patients exhibited positive results during treatment. T-cell proliferation tests were conducted on 25 patients; GV1001-specific T-cell proliferation was evident in 7 (28.0\%). The positive result of one patient is shown in Figure 2. Neither the ORR ( $42.8 \%$ in the positive vs. $53.3 \%$ in the negative group, $p=0.943$ ) nor the DCR $(100.0 \%$ in the positive vs. $88.9 \%$ in the negative group, $\mathrm{p}=1.000$ ) differed between the T-cell proliferation-positive and -negative groups. The median PFS (8.5 months [95\% CI, 3.0-13.9 months] vs. 4.7 months [ $95 \% \mathrm{CI}, 2.5-6.9$ months], $\mathrm{p}=0.303$ ) tended to be longer in the T-cell proliferation-positive group, but this difference was not statistically significant. The median OS could not be analyzed in this subgroup because of the censored data.

Table 4. Treatment-related adverse events (all patients, $n=56$ )

\begin{tabular}{lll}
\hline Treatment-related adverse events & \multicolumn{2}{l}{ All patients $(\mathrm{n}=56)$ : Number of patients $(\%)$} \\
\cline { 2 - 3 } Hematologic adverse events & All grade & $\geq$ Grade 3 \\
Leukopenia & $18(32.1 \%)$ & $1(1.8 \%)$ \\
Neutropenia & $27(48.2 \%)$ & $9(16.1 \%)$ \\
Febrile neutropenia & $0(0.0 \%)$ & $0(0.0 \%)$ \\
Anemia & $24(42.9 \%)$ & $0(0.0 \%)$ \\
Thrombocytopenia & $10(17.9 \%)$ & $0(0.0 \%)$ \\
Non-hematologic adverse events & \\
Anorexia & $10(17.9 \%)$ & $0(0.0 \%)$ \\
Nausea & $15(26.8 \%)$ & $4(7.1 \%)$ \\
Vomiting & $8(14.3 \%)$ & $4(7.1 \%)$ \\
Stomatitis & $12(21.4 \%)$ & $0(0.0 \%)$ \\
Neuropathy & $14(25.0 \%)$ & $2(3.6 \%)$ \\
Diarrhea & $12(21.4 \%)$ & $1(1.8 \%)$ \\
Infection & $3(5.4 \%)$ & $1(1.8 \%)$ \\
Skin rash & $5(8.9 \%)$ & $0(0.0 \%)$ \\
AST/ALT increased & $3(5.4 \%)$ & $0(0.0 \%)$
\end{tabular}

\section{Discussion}

In this study, we evaluated the efficacy and safety of GV1001 combined with chemotherapy in CRCs patients. To our knowledge, this is the first study to test a telomerase vaccine in patients with CRC.
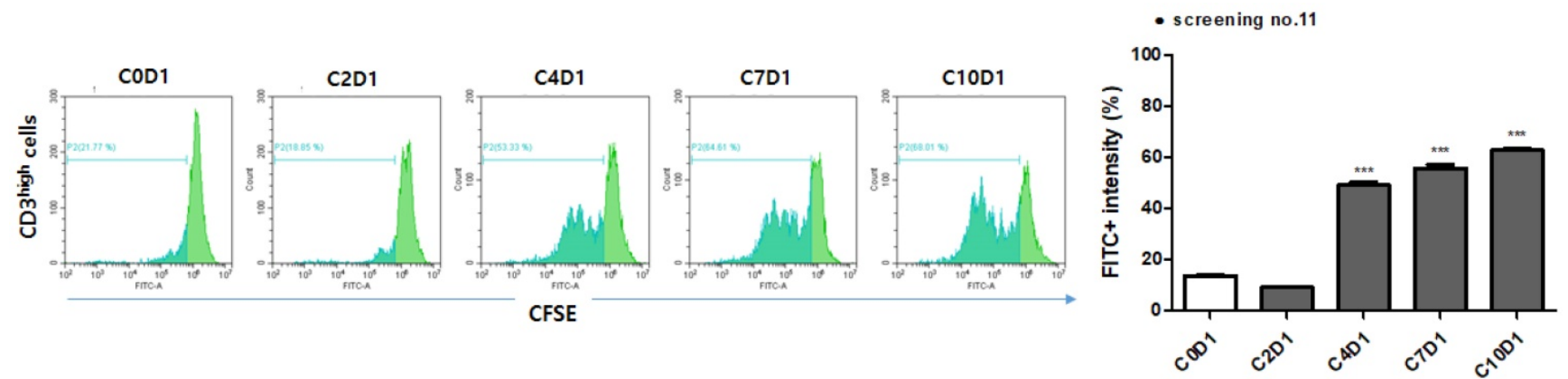

Figure 2. In vitro T cell proliferation in PBMC before the vaccination (COD1) and after GV1001 vaccination (cycles 2, 4, 7 and 10). Histogram plots showing the division peaks following anti-CD3 $(1 \mu \mathrm{g} / \mathrm{mL})$, anti-CD28 $(1 \mu \mathrm{g} / \mathrm{mL})$, and GV1001 $(20 \mu \mathrm{g} / \mathrm{mL})$ stimulation of carboxy fluorescein diacetate succinimidyl ester (CFSE)-labeled CD3high cells. Significance was evaluated by one-way ANOVA. $*^{*} \mathrm{p}<0.01$, and $* * * p<0.001$. In patient no. 11 , T cells started to divide (histogram) from the fourth cycle of the GV1001 vaccination, and the CFSE intensity of CD $3^{\text {high }}$ cells also significantly increased from the fourth cycle to the tenth cycle of treatment. 
In the results, the DCR was $90.9 \%$ (95\% CI, 82.4-99.4); this was higher than the predefined value for proof of efficacy. The median PFS and OS (secondary endpoints) were 7.1 months (95\% CI, 5.29.1 months) and 12.8 months (95\% CI, 9.9-15.8 months); these were comparable to the values in pivotal studies of second-line chemotherapies for CRCs [21, 22]. However, no obvious immune response (on the DTH or T-cell proliferation test) was observed, in contrast to other GV1001 trials with pancreatic and non-small cell lung cancers. No patient exhibited positive results on the DTH test; antigen-specific T-cell proliferation was observed in only $28 \%$ of patients ( 7 of 25 ). In addition, the results of the T-cell proliferation test were not correlated with the efficacy outcomes. A discrepancy between the DTH reactions and T-cell responses was also observed in a previous study on pancreatic cancer patients [12] and a plausible explanation is suggested that different sensitivities in the two assays or biologically different immune reactions generated by vaccination might have influenced the outcomes. Considering our results, it is difficult to clearly determine whether the observed efficacy is attributable to the synergistic effect of GV1001 vaccination and chemotherapy or to chemotherapy itself.

Unlike other studies of GV1001, we did not combine injections of granulocyte macrophage colony-stimulating factor (GM-CSF) with GV1001 vaccination, which may explain why we did not observe an obvious immune response. In general, a level of immunogenicity that breaks the immune tolerance of the host is essential for a cancer vaccine to be effective; concomitant delivery of adjuvant GM-CSF with a vaccine is widely adopted strategy. Although GM-CSF-based vaccines induced potent anti-tumor immune responses in preclinical studies $[23,24]$, the effects were not robust in clinical trials; they sometimes contradicted the results from animal models [25, 26]. Under certain conditions, GM-CSF induces the production of myeloid-derived suppressor cells and immunosuppressive regulatory $T$ cells, leading to unexpected outcomes. Based on the fact that adding GM-CSF to vaccination could induce immunosuppression [27], we hypothesized that GV1001 vaccination alone (i.e., without GM-CSF injection) might induce an adequate immune response. However, it is possible that the omission of GM-CSF may have compromised immunogenicity.

Moreover, when cytotoxic chemotherapy is used in conjunction with anti-cancer vaccination, the chemotherapy itself is immunosuppressive and can thus affect antigen-specific T-cell responses. Gemcitabine and fluorouracil, the combination partners of GV1001 in phase III trial of pancreatic cancer, have preclinical evidence for synergism with GV1001 that these agents induce apoptosis of cancer cells leading to the release of antigens which can be taken up by antigen-presenting cells and cross-presented to cytotioxic T cells [28, 29]. However, there is lack of evidence that oxaliplatin and irinotecan (the drugs used in the present study) act synergistically with GV1001.

Eotaxin is an eosinophil-specific chemokine associated with allergic reactions [30]. In general, chemokines have important roles in cancer progression that involve modulating tumor cell growth and migration [31]. However, little is known regarding the role of eotaxin in cancer. After the predictive value of eotaxin for GV1001 vaccination has been proposed in TeloVac study [15], consistent results were obtained in a subsequent phase III study of pancreatic cancer patients with high eotaxin levels [16]. However, in this study, there was no significant relationship between baseline eotaxin level and efficacy outcomes. Because no obvious immune response was induced, it is difficult to clearly explain a possible role for eotaxin as a predictive marker in this study. In addition, considering the conflicting results of the role of eotaxin in various types of cancer, the role of eotaxin as a predictive marker for GV1001 in pancreatic cancer as well as in other cancers needs to be further verified. Indeed, eotaxin level was associated with a poor prognosis in certain types of cancer [32]; conversely, it was associated with tumor suppression in other cancers [33].

\section{Conclusion}

Although no obvious immune response was observed, this first clinical study of telomerase vaccination for CRC patients showed that GV1001 vaccination in combination with conventional chemotherapy was tolerable and associated with modest efficacy outcomes. More robust studies are required to validate a potential role for GV1001 in CRC treatment.

\section{Supplementary Material}

Supplementary table. https://www.jcancer.org/v13p1363s1.pdf

\section{Acknowledgements}

This research was supported by the Hallym University Research Fund 2020 (HURF-2020-41), Anyang-si, Korea. GV1001 was generously provided by GemVax \& KAEL, Seongnam-si, Korea.

\section{Ethics statement}

The investigation was performed in accordance with the Declaration of Helsinki; the study protocol 
was approved by the institutional review board of Hallym University Medical Center, Anyang-si, South Korea.

\section{Competing Interests}

The authors have declared that no competing interest exists.

\section{References}

1. Bray F, Ferlay J, Soeriomataram I, et al. Global cancer statistics 2018: GLOBOCAN estimates of incidence and mortality worldwide for 36 cancers in 185 countries. CA Cancer J Clin. 2018; 68: 394-424.

2. Le DT, Uram JN, Wang H, et al. PD-1 blockade in tumors with mismatchrepair deficiency. N Engl J Med. 2015; 372: 2509-2520.

3. Shiu K-K, Andre T, Kim TW, et al. KEYNOTE-177: Phase III randomized study of pembrolizumab versus chemotherapy for microsatellite instability-high advanced colorectal cancer. J Clin Oncol. 2021; 39: 6-6.

4. Shulze T, Kemmner W, Weitz J, et al. Efficiency of adjuvant active specific immunization with Newcastle disease virus modified tumor cells in colorectal cancer patients following resection of liver metastases: results of a prospective randomized trial. Cancer Immunol Immunother. 2009; 58: 61-69.

5. Hazama S, Nakamura Y, Tanaka H, et al. A phase II study of five peptides combination with oxaliplatin-based chemotherapy as a first-line therapy for advanced colorectal cancer (FXV study). J Transl Med. 2014; 12:108.

6. Okuno K, Sugiura F, Inoue K, et al. Clinical trial of a 7-peptide cocktail vaccine with oral chemotherapy for patients with metastatic colorectal cancer. Anticancer Res. 2014; 34: 3045-52.

7. Vasef MA, Ross JS, Cohen MB. Telomerase activity in human solid tumors. Diagnostic utility and clinical applications. Am J Clin Pathol. 1999; 112: S68-75.

8. Gunes C, Rudolph KL. The role of telomeres in stem cells and cancer. Cell. 2013; 152: 390-393.

9. Shay JW, Wright WE. Senescence and immortalization: role of telomeres and telomerase. Carcinogenesis. 2005; 26: 867-874.

10. Bertorelle R, Rampazzo E, Pucciarelli S, et al. Telomeres, telomerase and colorectal cancer. World J Gastroenterol. 2014; 20: 1940-1950.

11. Mocellin S, Pooley KA, Nitti D. Telomerase and the search for the end of cancer. Trends Mol Med. 2013; 19: 125-133.

12. Bernhardt SL, Gjertsen MK, Trachsel S, et al. Telomerase peptide vaccination of patients with non-resectable pancreatic cancer: A dose escalating phase I/II study. Br J Cancer. 2006; 95: 1474-1482.

13. Brunsvig PF, Aamdal S, Gjertsen MK, et al. Telomerase peptide vaccination: a phase I/II study in patients with non-small cell lung cancer. Cancer Immunol Immunother. 2006; 55: 1553-1564.

14. Middleton G, Silcocks P, Cox T, et al. Gemcitabine and capecitabine with or without telomerase peptide vaccine GV1001 in patients with locally advanced or metastatic pancreatic cancer (TeloVac): an open-label, randomised, phase 3 trial. Lancet Oncol. 2014; 15: 829-840.

15. Neoptolemos JP, Greenhalf W, Cox TF, et al. Predictive cytokine biomarkers for survival in patients with advanced pancreatic cancer randomized to sequential chemoimmunotherapy comprising gemcitabine and capecitabine (GemCap) followed by the telomerase vaccine GV1001 compared to concurrent chemoimmunotherapy in the TeloVac phase III trial. J Clin Oncol. 2014; 32: 4121-4121.

16. Jo JH, Kim Y-T, Choi HS, et al. KG 4/2015: A randomized, controlled, multicenter, open-label phase III clinical trial of GV1001 with gemcitabine/ capecitabine in previous untreated, eotaxin-high patients with advanced pancreatic ductal adenocarcinoma. J Clin Oncol. 2021; 39: 4020-4020.

17. Kyte JA, Gaudernack G, Dueland S, et al. Telomerase peptide vaccination combined with temozolomide: a clinical trial in stage IV melanoma patients. Clin Cancer Res. 2011; 17: 4568-4580.

18. Kokhaei P, Palma M, Hansson L, et al. Telomerase (hTERT 611-626) serves as a tumor antigen in B-cell chronic lymphocytic leukemia and generates spontaneously antileukemic, cytotoxic T cells. Exp Hematol. 2007; 35: 297-304.

19. Schlapbach C, Yerly D, Daubner B, et al. Telomerase-specific GV1001 peptide vaccination fails to induce objective tumor response in patients with cutaneous T cell lymphoma. J Dermatol Sci. 2011; 62: 75-83.

20. Bertorelle R, Briarava M, Rampazzo E, et al. Telomerase is an independent prognostic marker of overall survival in patients with colorectal cancer. $\mathrm{Br} \mathrm{J}$ Cancer. 2013; 108: 278-284.

21. Van Cutsem $E$, Tabernero $J$, Lakomy $R$, et al. Addition of aflibercept to fluorouracil, leucovorin, and irinotecan improves survival in a phase III randomized trial in patients with metastatic colorectal cancer previously treated with an oxaliplatin-based regimen. J Clin Oncol. 2012; 30: 3499-3506.

22. Bennouna J, Hiret S, Bertaut A, et al. Continuation of bevacizumab vs cetuximab plus chemotherapy after first progression in KRAS wild-type metastatic colorectal cancer: The UNICANCER PRODIGE18 randomized clinical trial. JAMA Oncol. 2019; 5: 83-90.

23. Zhu YT, Zhao Z, Fu XY, et al. The granulocyte macrophage-colony stimulating factor surface modified MB49 bladder cancer stem cells vaccine against metastatic bladder cancer. Stem Cell Res. 2014; 13: 111-122.
24. Chen G, Gupta R, Petrik S, et al. A feasibility study of cyclophosphamide, trastuzumab, and an allogeneic GM-CSF-secreting breast tumor vaccine for HER2+ metastatic breast cancer. Cancer Immunol Res. 2014; 2: 949-961.

25. Lassi K, Dawson NA. Update on castrate-resistant prostate cancer: 2010. Curr Opin Oncol. 2010; 22: 263-267.

26. Mitchell DA, Sayour EJ, Reap E, et al. Severe adverse immunologic reaction in a patient with glioblastoma receiving autologous dendritic cell vaccines combined with GM-CSF and dose-intensified temozolomide. Cancer Immunol Res. 2015; 3: 320-325.

27. Kusmartsev S, Gabrilovich DI. Immature myeloid cells and cancer-associated immune suppression. Cancer Immunol Immunother. 2002; 51: 293-298.

28. Nowak AK, Lake RA, Marzo AL, et al. Induction of tumor cell apoptosis in vivo increases tumor antigen cross-presentation, cross-priming rather than cross-tolerizing host tumor-specific CD8 T cells. J Immunol. 2003; 170: 4905-4913.

29. Galetto A, Buttiglieri S, Forno S, et al. Drug- and cell-mediated antitumor cytotoxicities modulate cross-presentation of tumor antigens by myeloid dendritic cells. Anticancer Drugs. 2003; 14: 833-843.

30. Ying S, Meng Q, Zeibecoglou K, et al. Eosinophil chemotactic chemokines (eotaxin, eotaxin-2, RANTES, monocyte chemoattractant protein-3 (MCP-3), and MCP-4), and C-C chemokine receptor 3 expression in bronchial biopsies from atopic and nonatopic (Intrinsic) asthmatics. J Immunol. 1999; 163: 6321-6329.

31. Balkwill F. Cancer and the chemokine network. Nat Rev Cancer 2004; 4: 540-550.

32. Horiuchi K, Mishima K, Ohsawa M, et al. Prognostic factors for welldifferentiated squamous cell carcinoma in the oral cavity with emphasis on immunohistochemical evaluation. J Surg Oncol. 1993; 53: 92-96.

33. Tepper RI, Coffman RL, Leder P. An eosinophil-dependent mechanism for the antitumor effect of interleukin-4. Science. 1992; 257: 548-551. 